\title{
Dislocated Extension - Type Supracondylar Humerus Fractures in Children: Single Centre Experience
}

\author{
Lazar Todorovic, Mile Petrovski, Aco Dimov, Riste Simeonov, Marjan Kamiloski, Lazo Jovcheski, Marko Spasov, Vesna \\ Cvetanovska \\ University Clinic for Pediatric Surgery, Faculty of Medicine, Ss Cyril and Methodius University of Skopje, Republic of \\ Macedonia
}

\author{
Citation: Todorovic L, Petrovski M, Dimov A \\ Simeonov R, Kamiloski M, Jovcheski L, Spasov \\ $M$, Cvetanovska V. Dislocated Extension - Type \\ Supracondylar Humerus Fractures in Children \\ Single Centre Experience. OA Maced J Med \\ Sci. $2014 \quad$ Mar 15; 2(1):29-33 \\ http://dx.doi.org/10.3889/oamjms.2014.005 \\ Key words: supracondylar humerus fractures: \\ closed reposition; percutaneous fixation; \\ children; retrospective analysis. \\ "Correspondence: Lazar Todorović, MD. \\ University Clinic for Pediatric Surgery, Faculty of \\ Medicine, Ss Cyril and Methodius University of \\ Skopje, Vodnjanska 17, 1109 Skopje, Republic \\ of Macedonia. \\ E-mail: todoroviclazar@yahoo.com \\ Received: 13-Nov-2013; Revised: 02-Dec- \\ 2013; Accepted: 03-Dec-2013; Online first: \\ 11-Dec-2013 \\ Copyright: () 2013 Todorovic L et al. This is \\ an open-access article distributed under the \\ terms of the Creative Commons Attribution \\ tere, which permits unrestricted use, \\ distribution, and reproduction in any use, \\ provided the \\ credited. \\ Competing Interests: The authors have \\ declared that no competing interests exist.
}

\begin{abstract}
Aim: The aim of this study is to show our experience in cases of dislocated extension type supracondylar humeral fractures and evaluate the results of their treatment in the period from 2000 to 2010 with a follow up of a maximum six months.

Material and Methods: A retrospective analysis of the clinical cases for the period $2000-2010$ was done. Children from 4 to 14 years of age with supracondylar fractures of the humerus extension type are included. The total number of patients treated at the Clinic for Pediatric Surgery was 230 .

Results: The results of the treatment were evaluated after the last control examination six months later. We noticed postoperative neurological damage in 10 cases (4.85\%). In 14 patients (6.8\%) we noticed long term deformities such as cubitus varus or cubitus valgus. Infection at the entering place of the needles occurred in 4 patients $(1.94 \%)$, which did not indicate premature extraction of the needles and conversion of the treatment.
\end{abstract}

Conclusion: As a conclusion, we can stress that our outcomes in treatment of dislocated suparacondylar fractures of the humerus in children meet the world standards.

\section{Introduction}

Supracondylar humeral fractures are common and represent about $15 \%$ of all fractures in children. Most of all are extension type fractures.

Suggested method of treatment of undislocated Gartland type I fractures is cast immobilization during a period of 3-4 weeks, followed by a period of physical rehabilitation. Bigger therapeutic challenge are Gartland type II and type III fractures, where standard therapeutic approach is closed reduction with percutaneous pinning with Sommer pins [1-5]. Open reduction is rarely necessary. It is used only in cases where closed reduction is impossible, in open fractures or in cases with neurovascular deficit. These fractures should be promptly reduced and stabilized, in order to prevent further neurovascular damage or other complications [6-8]. In modified Gartland classification there is type IV fracture with complete dislocation and instability in all directions [9].

The aim of this study is to show our experience with dislocated supracondylar fractures of the humerus - extension type, with report of the results of the treatment in the period $2000-2010$ with 
a follow up of minimum 6 months (Fig. 1).

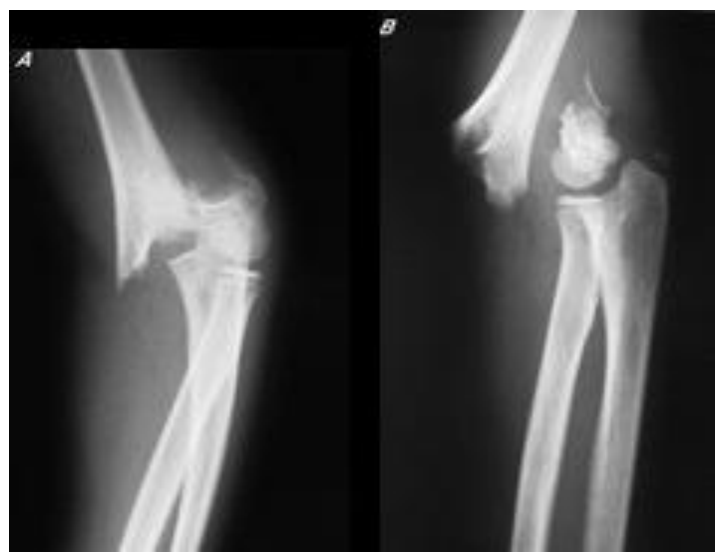

Figure 1: X-ray on admition.

\section{Material and Method}

A retrospective analysis of the clinical cases for the period $2000-2010$ was done. Children from 4 to 14 years of age with supracondylar fractures of the humerus - extension type are included. From the patients' files information about their age, mechanism of the injury, time from the injury to the admission and operation, method of the stabilization etc. was collected.

In the University Pediatric Surgery Clinic these fractures are treated promptly on the day of the admission [10, 11]. We prefer the method of closed reduction and percutaneous fixation with Sommer pins, in order to reduce the duration of the hospitalization, use of antibiotics and other drugs, to reduce the complications, and overall cost.

\section{Surgical technique}

In general anesthesia the patient lies on prone position [12]. Patient is moved close to the edge of the operation table.

Below the patient radio transparent board is placed which gives support to the injured elbow, in a manner where the forearm hangs freely and moves freely in all directions. Whole injured limb is scrubbed and prepped. The fluoroscope is also prepped and placed $90^{\circ}$ to the injured arm, lengthwise to the operation table.

Reduction is gained by longitudinal traction and direct pressure on the olecranon. The gravity force helps the reduction (Fig. 2, Fig. 3).

The position of the fragments is checked by fluoroscopy. All kinds of malrotation or inappropriate position are corrected by moving the distal fragment indirectly by moving the forearm. This is very important detail for prevention of postoperative deviations of the longitudinal axis of the arm in respect of varus of valgus position of the forearm.

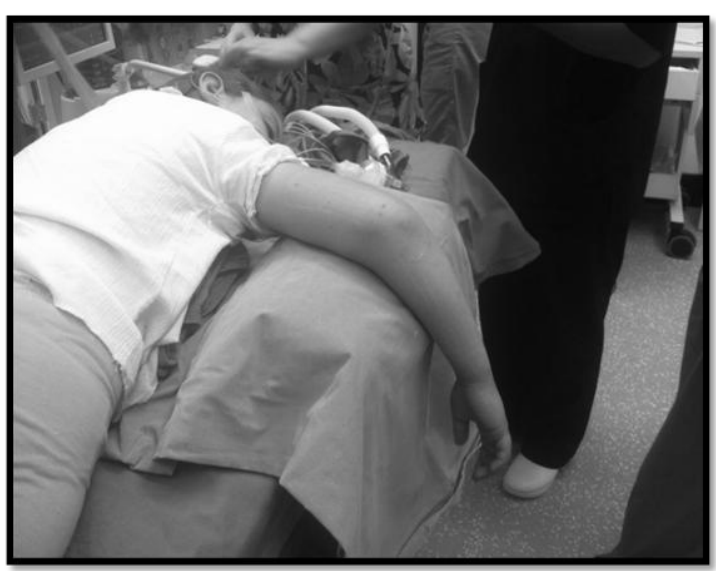

Figure 2: Positioning of the patient.

When the acceptable position is achieved, stabilization with Sommer pins is done. Dimensions of the pins are selected according the age and stature of the patient. By palpating the prominent spots of the elbow the penetrating points are selected and checked with antero-posterior x-ray.

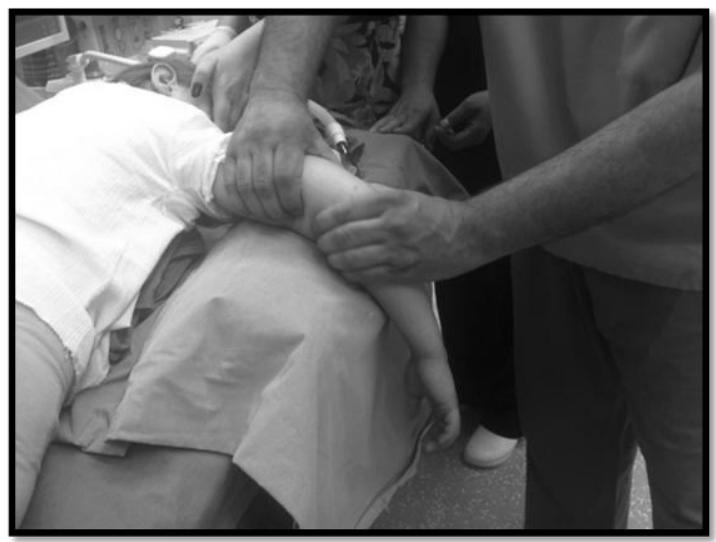

Figure 3: Closed reduction.

With fluoroscope control, two inserted pins have to end in the opposite cortex of the humerus. Profile control is mandatory, to avoid injury of the structures passing the anterior side of the humerus. In our institution the fracture is stabilized both from the medial and lateral side (Fig. 4).

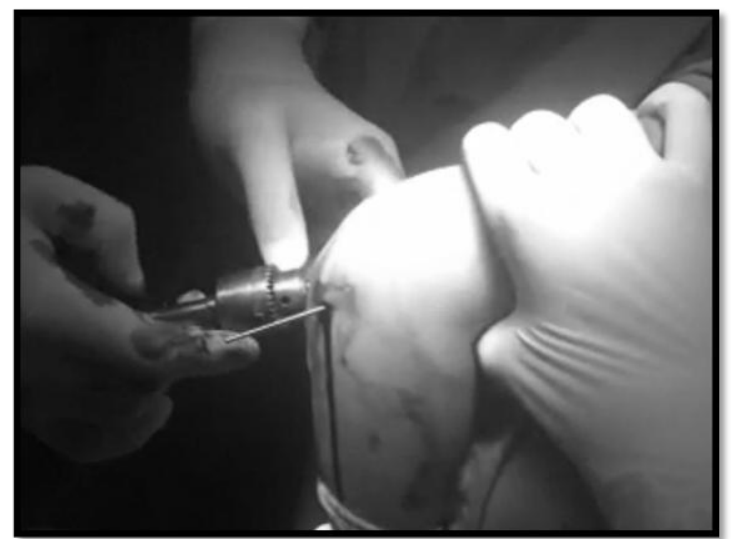

Figure 4: Pinning the lateral and medial humeral condyle using $T$ handle. 
The pin from the medial side is inserted very carefully, because of the anatomical position of the ulnar nerve. Some authors do not recommend pinning from the medial side due to the risk of damaging the ulnar nerve [13]. The pins are shortened and the ends twisted.

At the end, high cast immobilization is placed, which is removed along with the pins $3^{\text {rd }}$ or the $4^{\text {th }}$ week after the operation in outpatient, without the need of additional anesthesia or other procedures (Fig. 5).
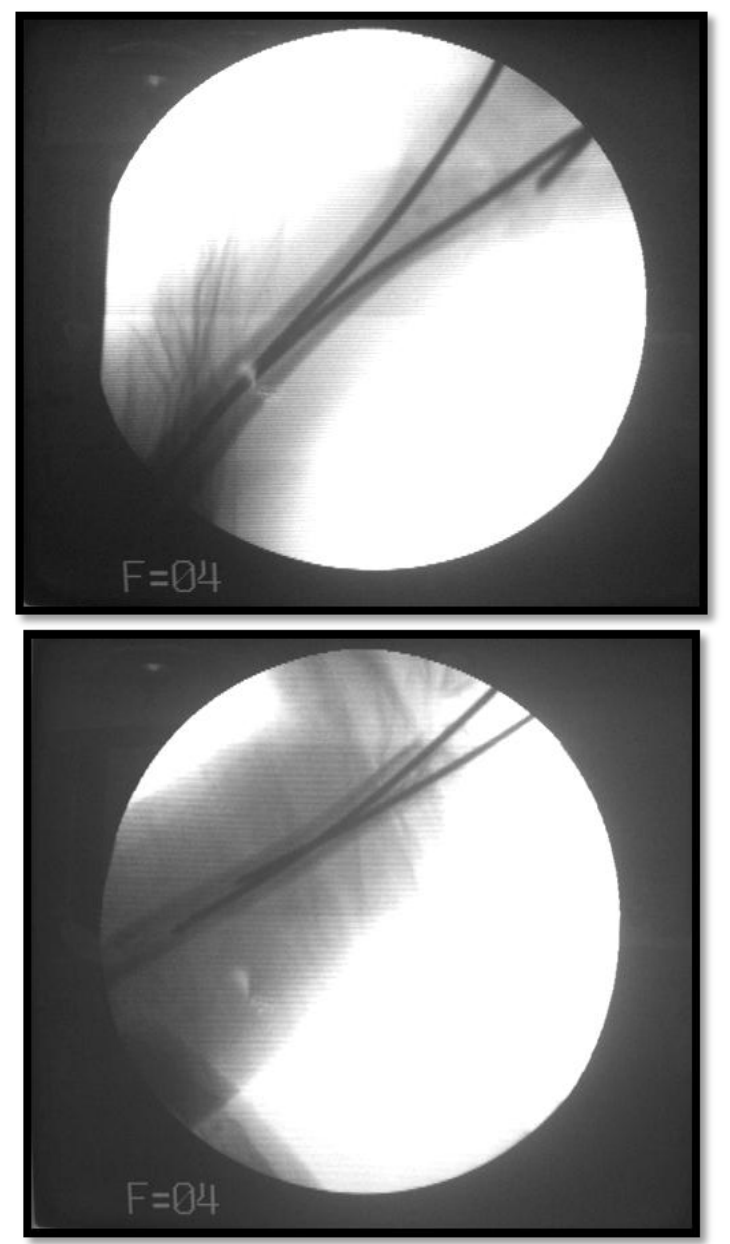

Figure 5: X-ray verification: adequacy of reduction and pin position.

\section{Results}

Total number of patients treated in The Clinic for pediatric surgery in the interval $2000-2010$ was 230. One hundred eighty two of them were dislocated Gartland type II and III. Twenty four of them were excluded from this study because they did not make checkup in the follow up period or had incomplete data in the history. There is not significant gender difference. The average age is 6.9 years.

From the patients' files data about the mechanism of the injury was gathered. Predominant mechanism is fall with extended elbow. The injuries in car crash are rare, and usually they are associated with other injuries.

In $6(2.91 \%)$ of the patients with Gartland type III fractures there was a vascular deficiency at the time of admission. After the immediate reposition, arterial pulsation distally of the injury was noticed, without a need of a further diagnostics or exploration of the vascular system. After the x-ray investigation, the fractures were classified according to Gartland.

The subjects of this study are Gartland type II and III fractures. Gartland type II was diagnosed in 64 patients $(31.07 \%)$, whereas Gartland type III was diagnosed in 118 patients $(57.28 \%)$. These fractures were treated in three ways: conservative method with closed reduction and cast immobilization, closed reduction with lateral and medial pinning with Sommer pins or open reduction with pinning.

Most of the surgical operations, where necessary, were performed on the day of the admission, or the next day, but not later than 24 hours after the injury. The results of the treatment are evaluated on the last check up after 6 months.

Aesthetic results are excellent in all patients. The end functional results were evaluated according to the measurement of the angular deformities, measurement of the girth of the $\operatorname{limb}$ and measurement of the range of the motion in the elbow joint $\left(0-135^{\circ}\right)$ (Fig. 6).

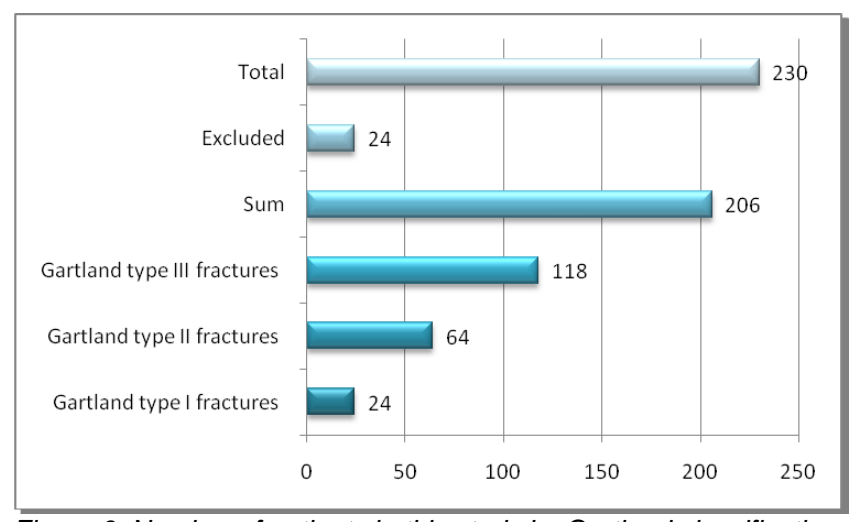

Figure 6: Number of patients in this study by Gartland classification.

Postoperative neurological deficit appeared in 10 cases $(4.85 \%)$, without a need of additional operative intervention. All neurological impairments after 6 months have resolved spontaneously. In 14 $(6.8 \%)$ long term problems as a consequence of bad reduction appeared. Cubitus varus - medialisation of the forearm in $10(4.85 \%)$ patients, whereas Cubitus Valgus - lateralisation of the forearm in the $4(1.94 \%)$ remaining patients.

These deformities were of such size that did not require additional intervention in respect of osteotomy and correction.

Infection in the entering spots of the pins appeared in 4 cases (1.94\%), without need of premature extraction of the pins and conversion of the treatment. 


\section{Discussion}

Extension type supracondylar fractures of the humerus are the most common fractures in childhood, thus have vast history of treatment. Nowadays different authors have different approach in solving these fractures by using many sorts of apparatuses for reposition, bone traction, and report comparative results with conventional way of treatment.

Speaking of conventional way of treatment, we point on 4 ways: conservative treatment with cast immobilization without reduction, conservative treatment with closed reduction and cast immobilization, closed reduction with percutaneous pinning with Sommer pins and open reduction and pinning.

Gartland type I fractures are treated with cast immobilization without reduction, followed by excellent results $[14,15]$.

In Gartland type II and III fractures, the therapy approach is controversial. Closed reduction and cast immobilization is indicated in Gartland type II fractures, but is considered inappropriate in Gartland type III fractures, because the incidence of secondary dislocation is high in these fractures.

Primary closed reduction with percutaneous pinning is method of choice for the Gartland type III fractures. But, we use this method in type II fractures also, to reduce the incidence of secondary dislocation and the need of additional $\mathrm{x}$-ray exposure.

Speaking of reduction, most commonly used maneuver for reposition is longitudinal traction of the limb, correction of the rotation and angulation in coronary plane and hyperflection and pronation of the forearm. This procedure mainly is performed with patient lying - supine. In this position the fracture is fixated with percutaneous pinning with Sommer pins. Flaw of this method is that in maximal flection in younger patients there is frontal dislocation of the ulnar nerve with bigger chance of lesion of the nerve. In our clinic, patient in general anesthesia as placed on his stomach - prone, thus the forearm hangs freely on a special plate $90^{\circ}$ to the arm. This position has few advantages. Gravity forces help the reduction, and the need of hyperflexion is avoided, and sometimes there is no need for the assistant to hold the reposition additionally. In the same time, deformity in sagital plane is avoided. Without the need of hyperflexion additional compromise of the blood perfusion distally to the fracture is avoided, thus avoided additional tissue damage and damage to the neural and vascular structures. Above all dislocation of the ulnar nerve is avoided, thus reducing the possibility of injury of the nerve.

The manner of pinning is controversial. Percutaneous cross-pinning or pinning medially and laterally with one pin on each side has been proved with lots of biomechanical studies as most stable biomechanical construction [16]. This technique by Swenson despite good biomechanical qualities often has been criticized by many authors because of the big percentage of jatrogenic postoperative lesion of the ulnar nerve. The nerve even not directly damaged, often is contused, pulled and suffers from the local edema. During the insertion of the pins with great rotation velocity, the fascia is pulled which indirectly pulls and damages the nerve [17]. Some authors stress that during the maximal flexion of the elbow, the ulnar nerve is dislocated and prone to injury.

According to Rassol, the safety of the percutaneous pinning is correlated with the experience of the surgeon. The precise manipulation when placing the medial pin lowers the complications of the ulnar nerve in respect of delayed neuropathy.

To avoid any risk of damaging the ulnar nerve, many institutions use the lateral pinning technique, placing two pins, both from the lateral side. They are placed absolutely parallel to each other, in distance of more than $1 \mathrm{~cm}$. If placed closer, their effect of stabilization decreases rapidly, and this fixation acts as a fixation with single pin only. This has been explained by Judet, author of the method of single lateral pinning technique.

In cases where reduction cannot be achieved by closed maneuvers, the open reduction is required, where the risk of lesion of the ulnar nerve is lower, because it is exposed and protected.

There is a doubt whether the ends of the needles should be left outside the skin or should be placed deep under the skin. According to some authors pins absolutely have to be placed below the skin thus avoiding complications like infections on the insertion place of the pins. This complication is rare, but when appears it compromises the postoperative course and healing with very bad aesthetic and functional outcomes. But, at the same time this method requires general anesthesia for extraction of the pins which is considered a big flaw.

In the Pediatric surgery clinic, the ends of the pins, as described above, are twisted, cut, and left to stick above the skin. They are extracted in outpatient conditions without anesthesia, which is considered a quality.

In our series, infection of the insertion port was reported only in four cases, without compromise of the postoperative course. Premature extraction was not necessary.

As a conclusion, we can stress that our outcomes in treatment of dislocated suparacondylar fractures of the humerus in children meet the world standards. This method in out Institution is considered routine, performed daily by the on duty staff. The number of unsatisfying outcomes is minimal, and neither of them is causing major aesthetic and functional disorders. 


\section{References}

1. Arino VL, Lluch EE, Ramirez AM, Ferrer J, Rodrigez L, Baixauli F. Percutaneous Fixation of Supracondylar Fractures of the Humerus in Children. $J$ Bone Joint Surg Am. 1977;59:914-16.

2. Kanellopoulos AD, Yiannakopoulos CK. Closed Reduction and Percutaneous Stabilization of Pediatric T - Condylar Fractures of the Humerus. J Pediatr Orthop. Jan/Feb 2004; 24(1):13-16.

3. Mangwani J, Nadarajah R, Peterson JMH. Supracondylar Humeral Fractures in Children. Ten Years' Experience in a Teaching hospital. J.Bone Joint Surg. Br. 2006;88-B:362-5.

4. Mazda K, Boggione C, Fitousi F, Penneçot GF. Systematic Pinning of Displaced Extension - type Supracondylar Fractures of the Humerus in Children: A PROSPECTIVE STUDY OF 116 CONSECUTIVE PATIENTS. J.Bone Joint Surg. Br. Aug 2001; 83-B(6):888-93.

5. McLauchlan GJ, Walker CRC, Cowan B, Robb JE, Presxott RJ. Extension of the Elbow and Supracondylar Fractures in Children. J.Bone Joint Surg. Br. May 1999; 81-B(3):402-05.

6. Childress HM. Transarticular Pin Fixation in Supracondylar Fractures at the Elbow in Childen: A CASE REPORT. J.Bone Joint Surg. Am. 1972;54:1548-52.

7. Mehlman CT, Strub WM, Roy DR, Wall EJ, Crawford AH. The Effect of Surgical Timing on the Perioperative Complications of Treatment of Supracondylar Humeral Fractues in Children. J.Bone Joint Surg. Am. 2001; 83:323-27.

8. Scaggs $\mathrm{DI}$, Julia MH, Bassett J, Kaminsky C, Kay RM. Tolo VT, Operative Treatment of Supracondylar Fractures of the Humerus in Children: The Consequences of Pin Placement. J.Bone Joint Surg. Am. 2001; 83:735-40.

9. Leitch KK, Kay RM, Femino JD, Tolo VT, Storer SK, Skaggs DL. Treatment of Multidirectionally Unstable Supracondylar Humeral Fractures in Children. A Modified Gartland Type - IV Fracture. J.Bone Joint Surg. Am. 2006;88:980-85

10. Green NE. Editorial - Overnight Delay in the Reduction of Supracondylar Fractures of the Humerus in Children. J.Bone Joint Surg. Am. 2001;83:321-52.

11. Walmsely PJ, Kelly MB, Robb JE, Annan IH, Porter DE, Delay Increases the Need for Open Reduction of Type - III Supracondylar Fractures of the Humerus. J.Bone Joint Surg. Br. 2006; 88B:528-30.

12. Fowler TP, Marsh JL. Reduction and Pinning of Pediatric Supracondylar Humerus Fractures in the Prone Position. $J$ Orthop Trauma. 2006; 20:277-81.

13. Scaggs DL, Cluck MW, Mostofi A, Flynn JM, Kay RM, Lateral Entry Pin Fixation in the Management of Supracondylar Fractures in Children. J.Bone Joint Surg. Am. 2004; 86:70207.

14. Matsuzaki K, Nakatani N, Harada M, Tamaki T. Teratment of Supracondylar Fractures of the Humerus in Children by Skeletal Traction in a Brance. J.Bone Joint Surg. Br. March 2004; 86-B(2):232-38.

15. Zionts LE, McKellop HA, Hathaway R. Torsional Strength of Pin Configurations Used to Fix Supracondylar Fractures of the Humerus in Children. J. Bone Joint Surg. Am. 1994; 76:253-6.

16. Vlahović T, Bumči I. Biomechanical Evaluation of the Value of Osteosynthesis in Supracondylar Fracture of the Humerus Using Kirschner Pins In Children. Eur J Pediatr Surg. 2002;12:410-15

17. Culp RW, Osterman AL, Davidson RS, Skirven T, Bora FW. Neural injuries Assocoated with Supracondylar Fractures of the Humerus in Children. J.Bone Joint Surg. Am. 1990;72:1211-15. 